\title{
Circular dichroism calculation for natural products
}

\author{
Alfarius Eko Nugroho $\cdot$ Hiroshi Morita
}

Received: 20 February 2013/Accepted: 21 March 2013/Published online: 7 April 2013

(C) The Japanese Society of Pharmacognosy and Springer Japan 2013

\begin{abstract}
Determination of the absolute configuration (AC) is often a challenging aspect in the structure elucidation of natural products. When chiral compounds possess appropriate chromophore(s), electronic circular dichroism (ECD) may provide a powerful approach to the determination of their absolute configuration. Recently, ECD calculations by time-dependent density functional theory (TDDFT) have come to be used more commonly. In the present review, we give several examples of recent studies using TDDFT-calculated ECD spectra for the AC determination of natural products.
\end{abstract}

Keywords Circular dichroism - Absolute configuration · Density functional theory

\section{Introduction}

Determination of the absolute configuration (AC) of natural products often poses a challenging problem in structure elucidation. There are various approaches to the solution of the problem, e.g., X-ray crystallography, chiroptical methods, and NMR anisotropy methods, each with its own limitations. When chiral compounds possess appropriate chromophore(s), electronic circular dichroism (ECD) may provide a powerful approach to the determination of their AC. In general, AC determination using ECD compares the spectrum of a new compound having unknown $\mathrm{AC}$ to those of analogous compounds of known AC. However, AC determination by predicting the sign of one or more bands

A. E. Nugroho · H. Morita $(\bowtie)$

Faculty of Pharmaceutical Sciences, Hoshi University,

Ebara 2-4-41 Shinagawa-ku, Tokyo 142-8501, Japan

e-mail: moritah@hoshi.ac.jp in the ECD spectrum by using empirical, semi-empirical or non-empirical rules may be an option. Another option, which has become more widely used in the past 10 years, is to compare calculated and experimental ECD spectra.

ECD calculations have been used for the determination of ACs of natural products for decades, as seen in the $\pi$ SCF studies of ECD by Mason and coworkers [1]. However, its use was quite limited until the major advancement in ECD calculations of time-dependent density functional theory (TDDFT) was introduced, which resulted in a good compromise between computational costs and accuracy [210]. The principle of the determination of the ACs of natural products by ECD calculation is relatively simple: basically, calculated ECD spectra are compared with experimental ECD spectra. If the two data sets are very similar to each other, then highly reliable assignment is obtained. In the present review, we briefly explain the method of TDDFT calculation of ECD spectra and give several examples of its successful application to the determination of the $\mathrm{AC}$ of natural products, including our own previous works. There are hundreds of publications using TDDFT-calculated ECD spectra for the AC assignment of a broad range of compounds, and examples were selected from recently published studies, especially those from 2012.

\section{Computational methods}

ECD calculations generally involve two steps, first the conformational analysis of the compound to obtain the possible conformer(s), and second, the UV/ECD TDDFT calculation of each conformer(s). The conformational analysis is often done by Monte Carlo methods using molecular mechanics (MMFF94, etc.) and/or semi- 
Fig. 1 Steps for electronic circular dichroism (ECD) calculation by time-dependent density functional theory (TDDFT)
Structure of relative configuration

Conformational analysis by MM , Confirm by NMR data

Conformers

Geometry optimization by DFT

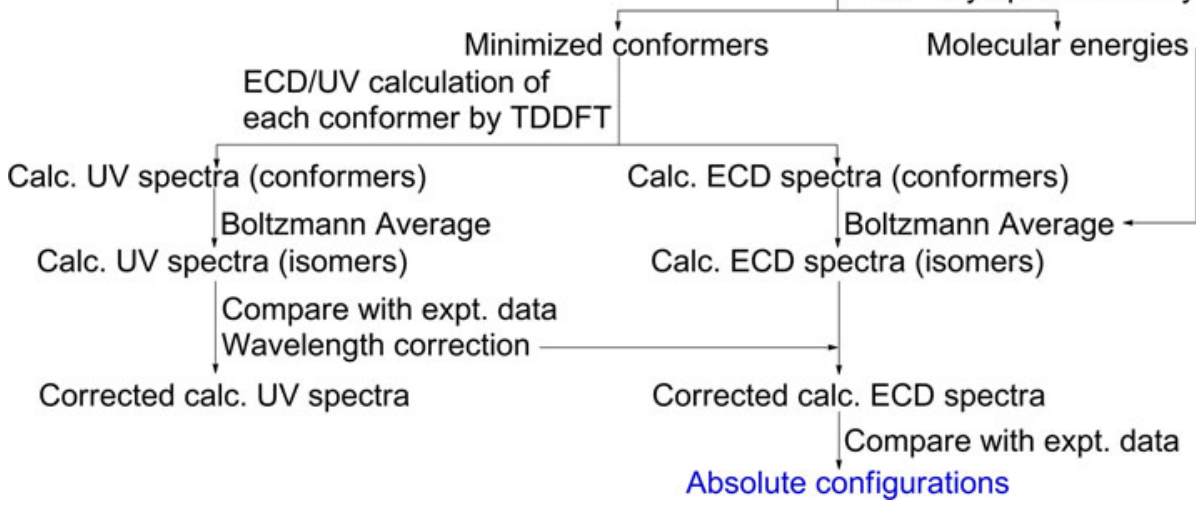

empirical methods (AM1, etc.) for the relative energy evaluation of the conformers. The resulting conformers are then optimized further using density functional theory (DFT) methods before being subjected to the TDDFT calculations of the UV/ECD by using programs such as Gaussian [11], TURBOMOLE [12], or NWChem [13]. To obtain the calculated UV/ECD spectra of the isomers, the UV/ECD spectra of the conformers are Boltzmann averaged. The averaged UV spectra are then shifted to conform to the experimental UV spectrum and the same shifts are also applied to the corresponding calculated ECD spectra before comparing the calculated ECD spectra with the experimental ECD of the natural product in question. The calculation steps are summarized in Fig. 1.

The accuracy of TDDFT calculations itself depends mainly on the basis set and functional used for the calculations. Use of a larger basis set generally increases the accuracy, but it also increases the computational time required. In ECD calculations, basis sets with polarization and diffuse functions, such as $6-31 G^{*}$ or aug-cc-pVDZ, and the B3LYP functional are commonly used and give satisfactory results [14]. The ECD calculation level is often expressed as [Functional 1]/[Basis set 1]//[Functional 2]/ [Basis set 2], which means that the ECD calculation was performed using Functional 1 and Basis set 1 on a geometry optimized by using Functional 2 and Basis set 2 .

The results of TDDFT calculations of UV/ECD are excitation energies, and their corresponding oscillator strength and rotatory strength. The oscillator strengths are used to simulate the UV curve, and rotatory strengths to simulate the ECD curve. Both oscillator strength and rotatory strength can be calculated using either dipolevelocity gauge or dipole-length gauge, but the use of dipole-length gauge generally gives better results [3, 15]. To take the solvent effect into account, a COnductor-like continuum Solvent MOdel (COSMO) can also be employed [16, 17].

The calculated rotatory strength values are generally converted to the line-shaped ECD curve using a Gaussian distribution function:

$\Delta \varepsilon(\sigma)=\frac{1}{2.296 \times 10^{-39}} \times \frac{1}{\Delta \sigma \sqrt{\pi}} \sum_{i} \sigma_{i} R_{i} e\left[\left\{-\frac{\sigma-\sigma_{i}}{\Delta \sigma}\right\}^{2}\right]$

where $\sigma$ is the wavenumber (in $\mathrm{cm}^{-1}$ ), $\Delta \sigma$ is half of the width of the band at $1 / e$ peak-height, and $\sigma_{\mathrm{i}}$ and $R_{\mathrm{i}}$ the excitation wavenumber and rotatory strength for transition $i$, respectively [18]. The value of $\Delta \sigma$ can be evaluated from the corresponding UV spectrum, and is typically in the range of $0.05-0.4 \mathrm{eV}$. The use of Lorentzian distribution to simulate ECD curves has also been reported by using the function:

$\Delta \varepsilon(\sigma)=\frac{1}{2.296 \times 10^{-39}} \times \frac{1}{\gamma \sqrt{\pi}} \sum_{i} \sigma_{i} R_{i}\left[\frac{\gamma^{2}}{\left(\sigma-\sigma_{i}\right)^{2}+\gamma^{2}}\right]$

where $\gamma$ represents half the width of the band at half peakheight [18-22].

\section{Use of TDDFT calculation of ECD spectra for the AC determination of natural products}

This section gives examples where the TDDFT calculation of ECD was used for the AC determination of natural products. The examples include alkaloids, terpenoids, coumarins, anthraquinones, and other types of compounds, ranging from conformationally rigid to highly flexible compounds. 
Fig. 2 Structure and selected ROESY (rotating-frame nuclear Overhauser effect correlation spectroscopy) correlations for eucophylline (1)

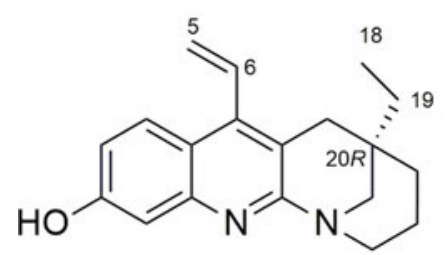

1

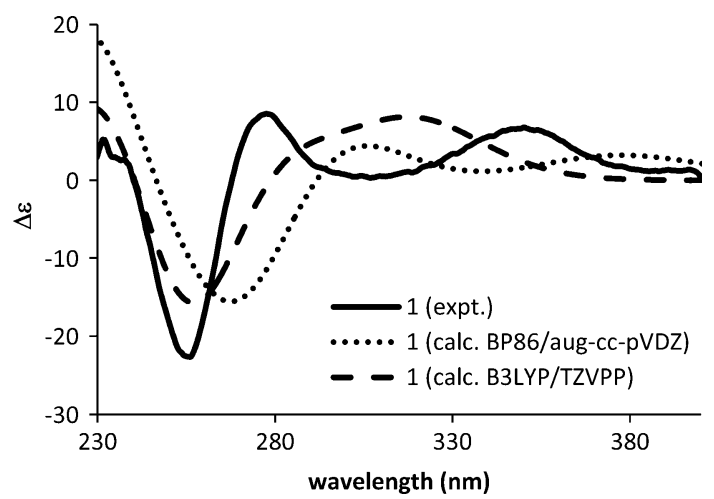

Fig. 3 Calculated $(20 R)$ and experimental ECD spectra of eucophylline (1)<smiles>CC[C@]12CCCN3C(=O)C(=O)OC31CC(=O)Nc1ccccc12</smiles><smiles>[R][C@]1(CC)CCCN2C(=O)c3[nH]c4ccccc4c3[C@H]21</smiles>

2 3: $\mathrm{R}=\mathrm{CH}_{2} \mathrm{CH}_{2} \mathrm{CO}_{2} \mathrm{Me}$

$$
3_{\text {dis }}: \mathrm{R}=\mathrm{Me}
$$

Fig. 4 Structures of leucomidines A (2) and B (3)

\section{Alkaloids}

\section{Eucophylline [23]}

Eucophylline (1), isolated from Leuconotis griffithii [23], has only one chiral center at C-20. Conformational analysis (MMFF94 force field, $50 \mathrm{~kJ} / \mathrm{mol}$ window) generated conformations that can be divided into two groups: one group with the piperidine ring in a chair form, and the other with the ring in a boat form. Members of the same group differ in the three-dimensional (3D) location of the ethyl moiety at $\mathrm{C}-20$ and the vinyl moiety at $\mathrm{C}-4$. The group with the piperidine ring in chair form was predicted to be dominant, which agreed with NMR data (Fig. 2). The ECD spectra

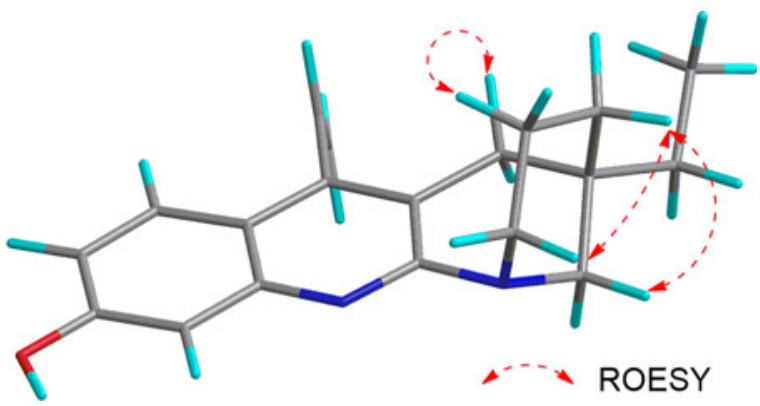

were calculated at the BP86/aug-cc-pVDZ//BP86/SVP and B3LYP/TZVPP//B3LYP/TZVPP level on two stable conformers differing in the location of the vinyl group because changes in the location of the ethyl moiety were expected to have no effect on the calculated ECD spectra. The ECD spectra for the two conformers were Boltzmann-averaged to obtain the ECD spectrum of the isomers. The two calculation levels lead to the same conclusion that the AC at $\mathrm{C}-20$ of 1 was $R$. Of the calculation levels, the BP86/augcc-pVDZ//BP86/SVP calculated ECD spectrum matched the experimental spectrum well, but in the B3LYP/ TZVPP//B3LYP/TZVPP calculated spectrum, the first and second cotton effects (CEs) apparently overlapped (Fig. 3).

\section{Leucomidines A and B [24]}

Leucomidines A and B (2 and 3, Fig. 4) are indole alkaloids also isolated from L. griffithii [24]. Conformational analysis (MMFF94 force field, $50 \mathrm{~kJ} / \mathrm{mol}$ window) for both 2 and 3 generated conformers differing only in the orientation of the side chain at C-20. The ECD spectra of the most stable conformation for $\mathbf{2}$ and $\mathbf{3}$ were each calculated at BP86/aug-cc-pVDZ//BP86/SVP and B3LYP/TZVPP// B3LYP/TZVPP levels. Both levels gave the same conclusion that both $\mathbf{2}$ and $\mathbf{3}$ had the $20 R, 21 S$ configuration. In the case of 2, however, the B3LYP/TZVPP//B3LYP/TZVPPcalculated ECD spectrum agreed with the experimental ECD spectrum better than that calculated by BP86/aug-ccpVDZ//BP86/SVP (Fig. 5). It is to be noted that the calculated ECD spectrum for $\mathbf{3}_{\text {dis }}$ (Fig. 5) - a model compound for $\mathbf{3}$ in which the side chain at $\mathrm{C}-20$ was substituted with a methylgroup-reproduced the experimental ECD spectrum better.

\section{Rupestines F-M [25]}

Rupestines F-M (4-11, Fig. 6) are guaipyridine alkaloids isolated from Artemisia rupestris [25]. Of those eight compounds, 5-11 showed the CE at about $270 \mathrm{~nm}$ in the experimental ECD spectra, which can be attributed to transition of the pyridine moiety. To study the effect of conformation on the CE sign at $270 \mathrm{~nm}$, the ECD spectra 

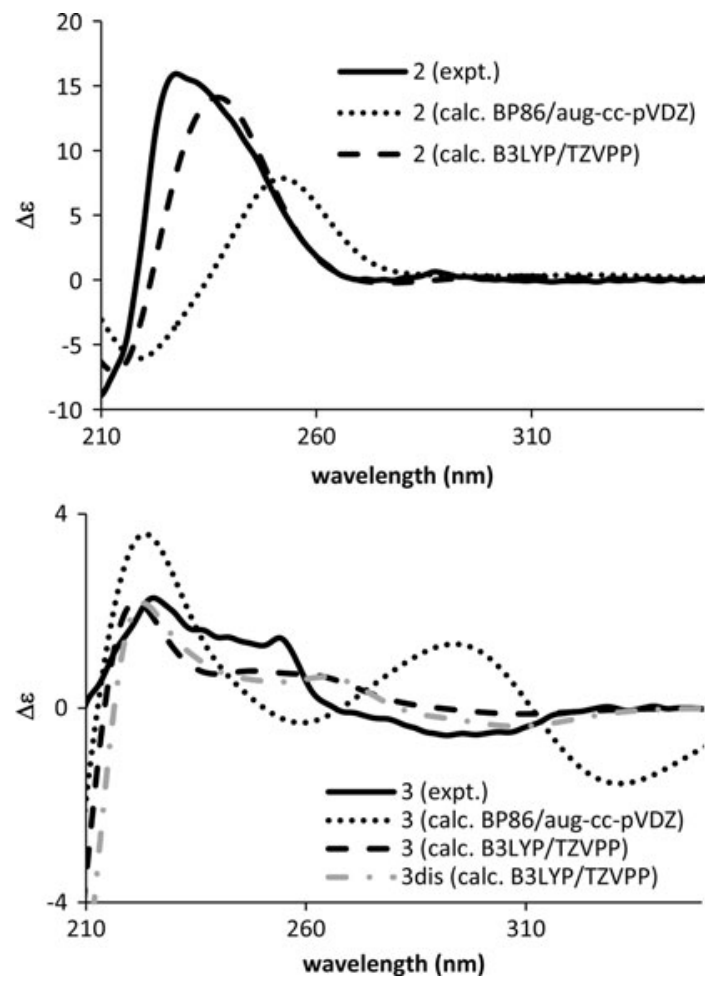

Fig. 5 Calculated $(20 R, 21 S)$ and experimental ECD spectra of leucomidines A (2) and B (3)

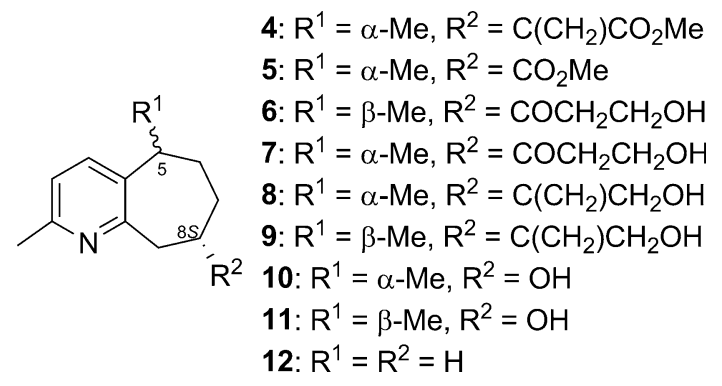

Fig. 6 Structures of rupestines F-M (4-11) and 2-methylpyridinecycloheptene (12)

of possible conformations of $\mathbf{1 0}, \mathbf{1 1}$ and a model compound, 2-methylpyridinecycloheptene (12), were calculated (Figs. 7, 8). The calculation results may be summarized as follows, (1) B3LYP/TZVPP calculations overestimated the excitation energy of pyridine's first absorption band (calc. $\sim 250 \mathrm{~nm}$ vs expt. $\sim 270 \mathrm{~nm}$ ), so that the calculated $\mathrm{CE}$ at around $250 \mathrm{~nm}$ are observed experimentally at around $270 \mathrm{~nm}$; (2) when the plane of C-6, C-7, and C-8 was over the pyridine ring, a positive CE at around $215 \mathrm{~nm}$ and a negative $\mathrm{CE}$ at around $250 \mathrm{~nm}$ were observed regardless of the chirality at C-5. Thus, by using the CE sign at around $270 \mathrm{~nm}$, the absolute structure of the molecules can be deduced or the AC at C-8 of 5-11 could be assigned accordingly; $\mathbf{5}-\mathbf{1 1}$ were shown to possess the same $8 S$ configuration.
The ECD spectrum of $\mathbf{4}$ is different from those of $\mathbf{5 - 1 1}$. By comparing the ECD spectrum calculated at B3LYP/ TZVPP//B3LYP/TZVPP level with the experimental spectrum (Fig. 9), the AC of $\mathbf{4}$ was assigned as $5 S, 8 S$.

\section{Bisnicalaterines B and C [26]}

Bisnicalaterines B and C (13 and 14) are rotamers isolated from Hunteria zeylanica [26]. By changing the dihedral angle $N-1-\mathrm{C}-16-\mathrm{C}-9^{\prime}-\mathrm{C}-10^{\prime}$, two stable conformations were obtained, one corresponding to the structure of $\mathbf{1 3}$ and the other to that of $\mathbf{1 4}$ (Fig. 11). Monte Carlo conformational analysis (MMFF94 force field, $50 \mathrm{~kJ} / \mathrm{mol}$ window) of each structure generated conformers differing only in conformation at C-20 (ethyl group, C-18 and C-19) and/or at $\mathrm{C}-15^{\prime}$ (hydroxyethyl group, $\mathrm{C}-16^{\prime}$ and $\mathrm{C}-17^{\prime}$ ), which should have little or no effect on the calculated ECD spectra. Thus, the ECD calculation was performed at BP86/ aug-cc-pVDZ//BP86/SVP level only for the most stable conformation of $\mathbf{1 3}$ and 14. The calculated and experimental ECD spectra for $\mathbf{1 3}$ and $\mathbf{1 4}$ are shown in Fig. 12, suggesting their AC to be as shown in Fig. 10.

\section{Schizozygine [27]}

Schizozygine (15) is an alkaloid isolated from Schizozygia caffaeoides [28]. Monte Carlo conformational search by using MMFF94 force field for the energy evaluation generated two stable conformations within the $20 \mathrm{kcal} / \mathrm{mol}$ window. The two conformations were then re-optimized at the B3LYP/6-31G* level, and the results were subjected to a potential energy scan (PES) at the B3LYP/6-31G* level. By varying the dihedral angle C-9-C-10-O-C-24 of both of the conformations, two additional conformations were identified. The ECD calculations for all four conformers were done at the B3LYP/aug-cc-pVDZ//B3LYP/6-31G*, B3LYP/aug-cc-pVDZ//B3LYP/TZ2P, and B3LYP/aug-ccpVDZ//B3PW1/TZ2P levels. For each conformer, calculations at all three levels gave similar results. The ECD spectra were Boltzmann-averaged, and the resulting ECD spectrum agreed well with the experimental ECD spectrum. The AC of $\mathbf{1 5}$ was assigned as shown in Fig. 13 [27].

\section{Actinophyllic acid [29]}

The AC of actinophyllic acid (16) —an alkaloid isolated from Alstonia actinophylla [30] —was assigned as shown in Fig. 13 indirectly on the basis of its methyl ester derivative because of the expected complication due to the presence of the carboxylic acid group [29]. A preliminary conformational search (MMFF94) gave two stable conformers within a $10-\mathrm{kcal} / \mathrm{mol}$ window. Of the ECD spectra calculated at B3LYP/6-31G(d,p)//B3LYP/6-31G(d,p) and 
Fig. 7 Calculated conformations of model compounds

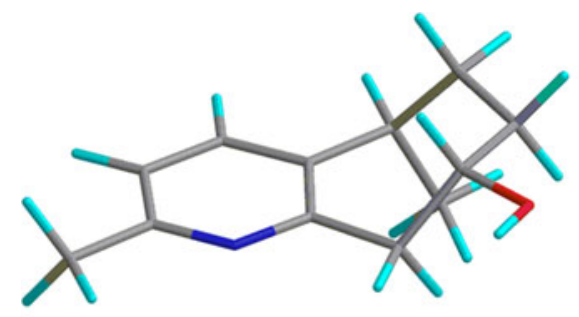

10

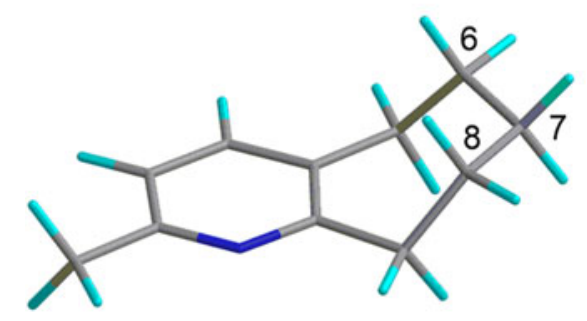

$12 a$

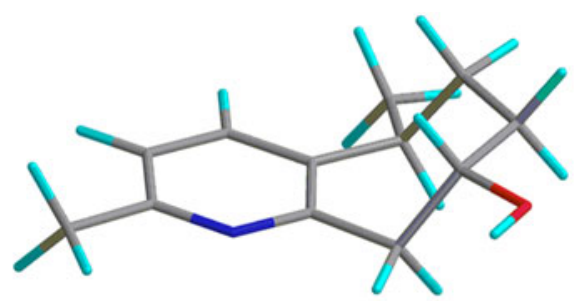

11
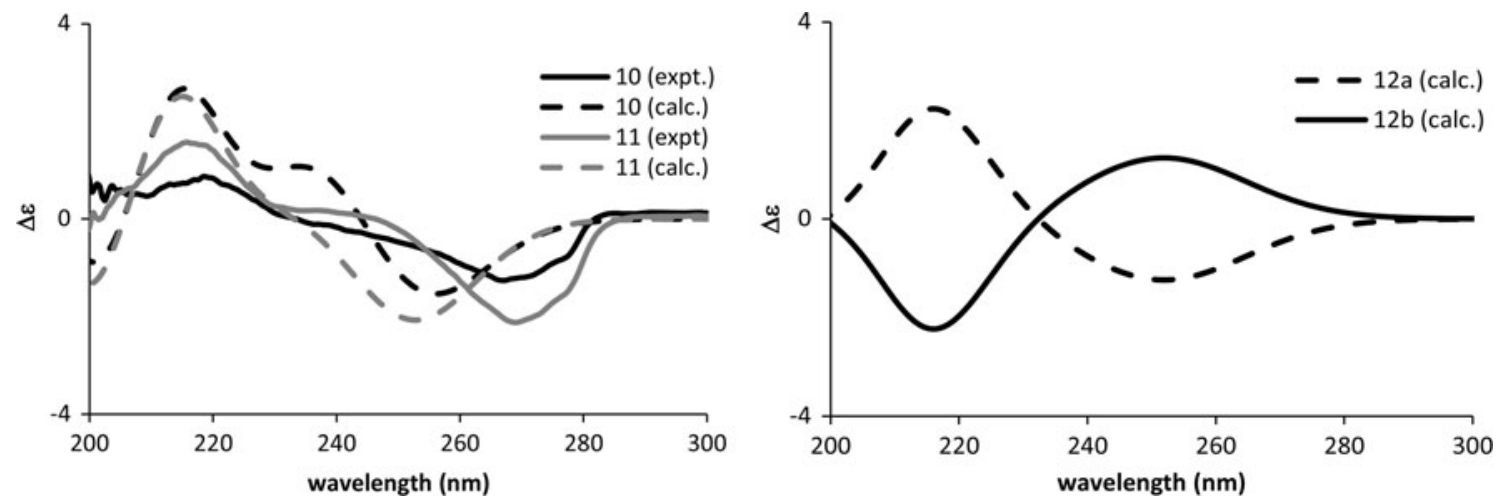

Fig. 8 Calculated circular dichroism (CD) spectra of model compounds together with the experimental CD spectra of rupestines L (10) and M (11)

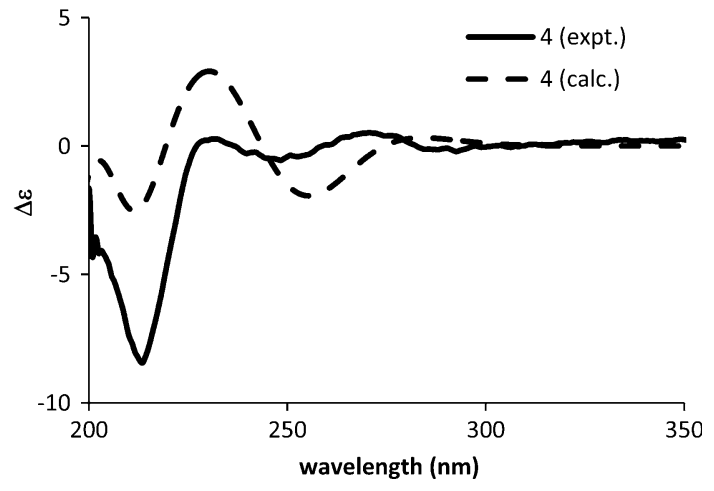

Fig. 9 Calculated $(5 S, 8 S)$ and experimental ECD spectra of rupestine $\mathrm{F}$ (4)

B3LYP/aug-cc-pVDZ//B3LYP/6-31G(d,p) levels, the one calculated at the B3LYP/6-31G(d,p) level generally agreed well with the experimental ECD spectrum, but

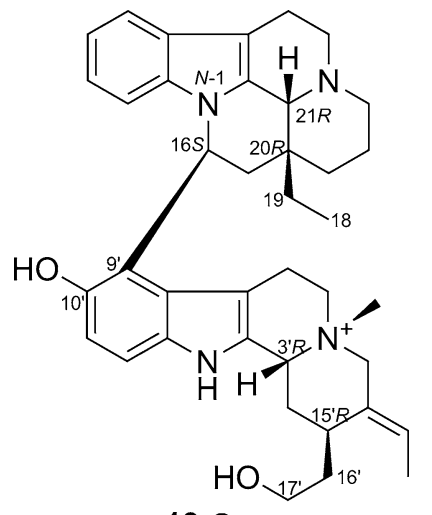

13: $S_{a}$

$14: R_{\mathrm{a}}$

Fig. 10 Structures of bisnicalaterines B (13) and C (14)

did not give the negative shoulder at around $230 \mathrm{~nm}$. On the other hand, the B3LYP/aug-cc-pVDZ-calculated ECD spectrum clearly reproduced the negative shoulder 
Fig. $113 \mathrm{D}$ structures of bisnicalaterines $\mathrm{B}(\mathbf{1 3})$ and $\mathrm{C}$ (14)

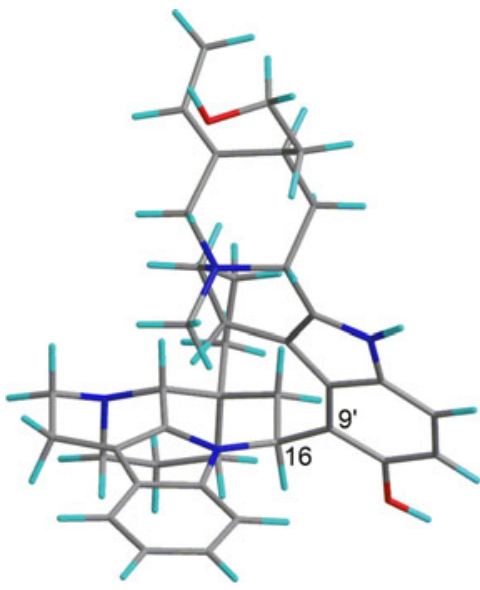

13

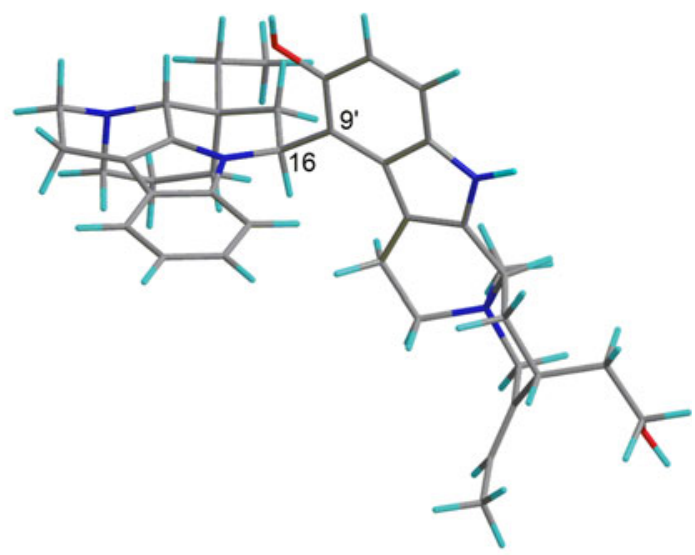

14

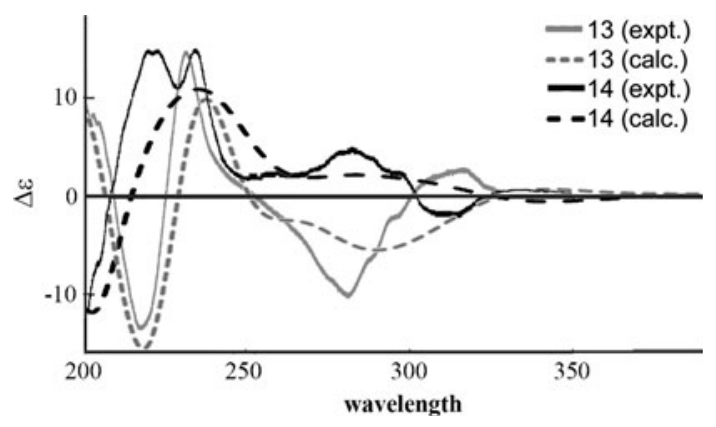

Fig. 12 Calculated $\left(16 S, 20 R, 21 R, 3^{\prime} R, 15^{\prime} R\right)$ and experimental ECD spectra of bisnicalaterines B (13) and C (14)

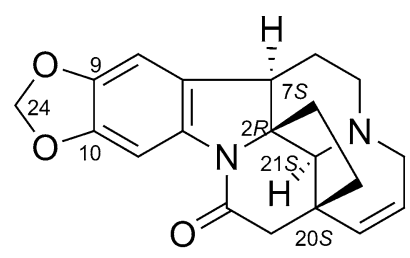

15

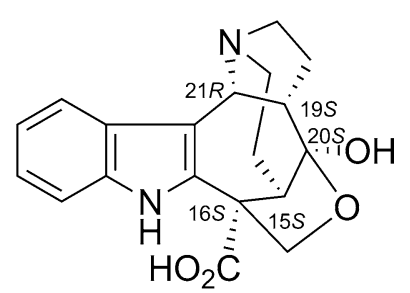

16
Fig. 13 Structures of schizozygine (15) and actinophyllic acid (16)

around $230 \mathrm{~nm}$, but the region around $250 \mathrm{~nm}$ became slightly positive.

\section{Mariline $A_{1}$ and $A_{2}[31]$}

Mariline $A_{1}(\mathbf{1 7})$ and $A_{2}$ are enantiomeric phthalimidines isolated from sponge-derived fungus Stachylidium sp. [31]. Detailed investigation of the highly flexible conformations of $\mathbf{1 7}$ was carried out at the B3LYP/SV(P) level, yielding more than 550 conformers. ECD calculations were performed at the B3LYP/SV(P) level with the COSMO model. Comparison of the calculated ECD spectra with the experimental spectrum did not allow an unambiguous assignment of the AC. Almeida and coworkers [31] associated the problem with dispersion effects caused by the highly flexible alkoxy side chain. Instead of using a dispersion-corrected functional in combination with a larger basis set to perform a DFT optimization of the conformations, they used single-point energy calculations for all previously DFT-optimized structures at the RI-SCS-MP2/ TZVPP level since a very accurate valuation of the energies for the Boltzmann weighting for such a high number of conformers was indispensable. Boltzmann weighting with these more reliable energy values yielded a significantly improved overall ECD spectrum, which almost agreed with the experimental one. Thus the AC of $\mathbf{1 7}$ was determined to be as shown in Fig. 14.

\section{Chaetoglobosin $V_{b}[32]$}

Chaetoglobosin $\mathrm{V}_{\mathrm{b}}(\mathbf{1 8})$-a cytochalasan alkaloid—was isolated from Chaetomium globosum [32]. Xue and coworkers performed a TDDFT ECD calculation [B3LYP/ 6-311 + G(d,p)//B3LYP/6-311 + G(d,p)] using a model compound of $\mathbf{1 8}$ in which the 3-methylindole moiety was replaced by a hydrogen atom. They considered that the 3 -methylindole ring should contribute only weakly to the observed ECD spectrum of $\mathbf{1 8}$ in the long-wavelength region, because (1) it is connected to the chiral skeleton by two flexible bonds; (2) it is quite distant from the other<smiles>COc1ccc(N2C(=O)c3c(cc(OC/C=C(\C)CCC=C(C)C)c(C)c3OC)[C@H]2C)c(CCC=C(C)C)c1</smiles>

Fig. 14 Structure of mariline $A_{1}(17)$ 


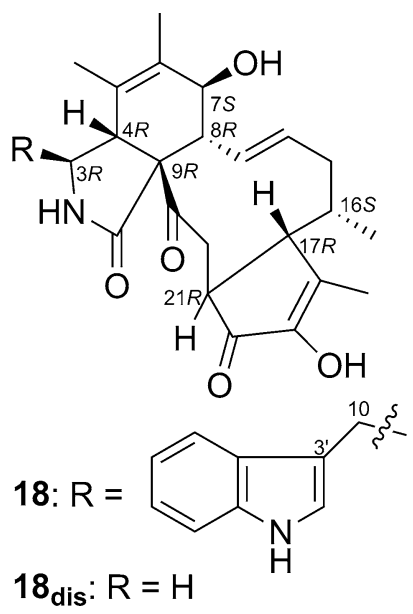

Fig. 15 Structure of chaetoglobosin $V_{b}(\mathbf{1 8})$

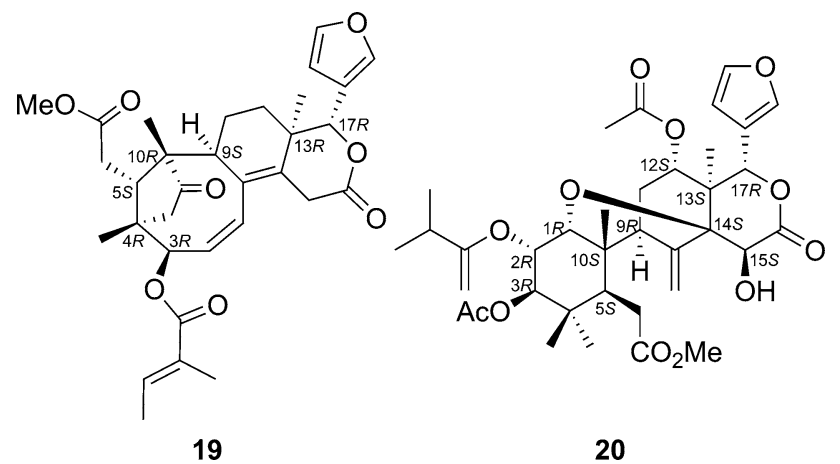

Fig. 16 Structures of chisomicine A (19) and sanjecumin B (20)

chromophores in the molecule, especially the cyclopentenone; and (3) its transitions above $220 \mathrm{~nm}$ are either very weak or directed roughly perpendicular to the C-3'/C-10 bond; so its coupling with other chromophores should be near zero. The use of this model compound simplified ECD calculations, since it gives only two conformers to be considered for the calculations. Based on the calculation results, the AC of $\mathbf{1 8}$ was suggested to be as shown in Fig. 15.

\section{Terpenoids}

\section{Chisomicine A [33] and sanjecumin B [34]}

Chisomicine A (19) [33] and sanjecumin B (20) [34] are limonoids isolated from Chisocheton ceramicus (Meliaceae), and from Sandoricum koetjape (Meliaceae), respectively. ECD calculations by TDDFT suggested the ACs of $\mathbf{1 9}$ and $\mathbf{2 0}$ to be as shown in Fig. 16. Calculations were performed at B3LYP/SVP//B3LYP/SVP level and at BP86/aug-cc-pVDZ//BP86/SVP level for chisomicine A and sanjecumin $\mathrm{B}$, respectively. The calculated ECD spectra are shown in Fig. 17 along with the experimentally obtained spectra.

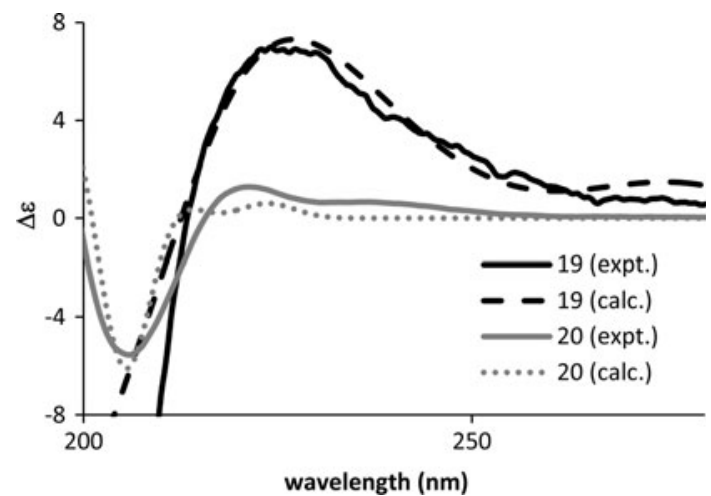

Fig. 17 Calculated $(17 R)$ and experimental ECD spectra of chisomicine A (19) and sanjecumin B (20)

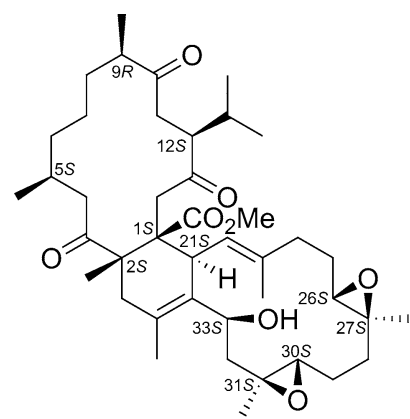

21

Fig. 18 Structure of ximaolide A (21)

\section{Ximaolides [35]}

Ximaolides, including ximaolide A (21, Fig. 18), are a series of bis-cembrane diterpenoids obtained from the Hainan soft coral Sarcophyton tortuosum [36, 37]. Kurtán and coworker [35] assigned the AC of $\mathbf{2 1}$ by comparing the solid-state ECD spectrum of $\mathbf{2 1}$ with the TDDFT-calculated ECD spectrum of the solid-state geometry obtained from X-ray crystallography analysis. By this method, they managed to reduce the number of input conformers for the ECD calculation of this highly flexible compound to only one, and thus the $\mathrm{AC}$ of $\mathbf{2 1}$ was determined as shown in Fig. 18.

\section{Coumarins}

\section{5-O-methyldiphysin and 5,5"-di-O-methyldiphysin [38]}

Bis-dihydrocoumarins, 5-O-methyldiphysin (22) [38] and 5,5"-di-O-methyldiphysin [39] were isolated from Ormocarpum kirkii. By using the non-empirical aromatic quadrant rule [40]. Dhooghe and coworkers [39] assigned the $\mathrm{AC}$ of $5,5^{\prime \prime}$-O-dimethyldiphysin to be of the 


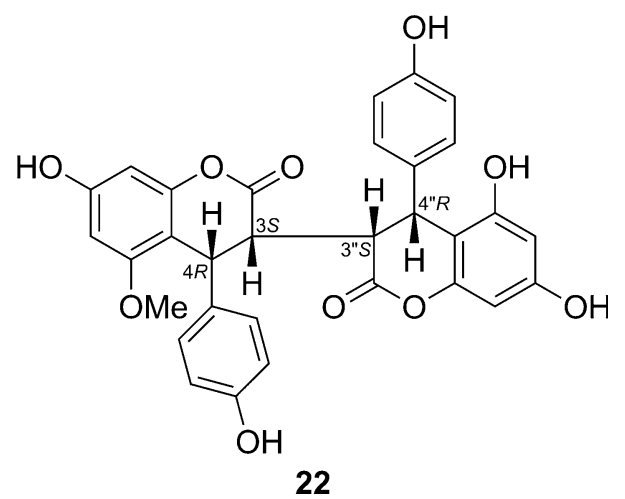

Fig. 19 Structure of 5-O-methyldiphysin (22)

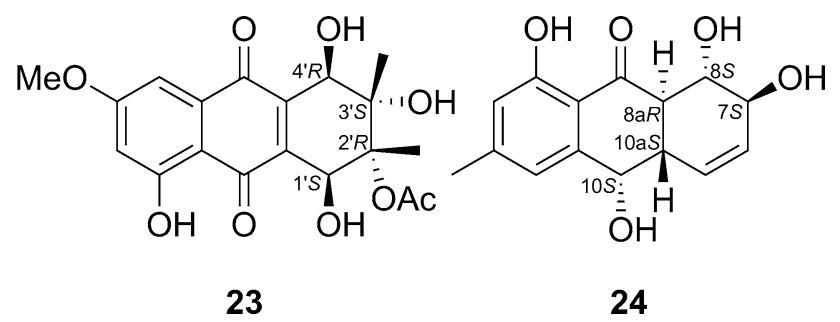

Fig. 20 Structures of altersolanol N (23) and coniothyrinone A (24)

$3 R, 4 S, 3^{\prime \prime} R, 4^{\prime \prime} S$ configuration. However, Xu and coworkers [38] showed that the TDDFT ECD calculation of $\left(3 S, 4 R, 3^{\prime \prime} S, 4^{\prime \prime} R\right)-22$ in both gas and solution phases [(B3LYP/6-31G(d,p)//B3LYP/6-31G(d,p)] produced an ECD spectrum quite similar to the reported experimental spectrum of 5,5"-di-O-methyldiphysin, indicating that the non-empirical aromatic quadrant rule is not appropriate for defining the AC of $\mathrm{C}-4$ of this class of compounds (Fig. 19).

\section{Anthraquinones}

\section{Altersolanol N [41]}

Altersolanol N (23) is an anthraquinone derivative isolated from Stemphylium globuliferum-an endophytic fungus isolated from Mentha pulegium (Lamiaceae) [41]. First, DFT minimizations were executed at B3LYP/6$31 \mathrm{G}(\mathrm{d})$ level, and all the resulting DFT minima with relative internal energies within $10 \mathrm{kcal} / \mathrm{mol}$ were reoptimized at the B3LYP/6-311G + (d,p) level with the polarizable continuum model (PCM) for acetonitrile. For the TDDFT ECD calculations, a preliminary screening of various functionals (B3LYP, CAM-B3LYP, BH\&HLYP, PBE0) and basis sets (SVP, TZVP, aug-TZVP) showed that CAM-B3LYP provided the best result, and the results obtained by the use of the smallest basis set used (SVP) was compatible with those obtained by the use of larger ones. Therefore, the CAM-B3LYP/SVP combination was chosen for the final calculations, which determined the AC of $\mathbf{2 3}$ to be as shown in Fig. 20.

Coniothyrinone A [42]

Coniothyrinone A (24), an anthraquinone derivative, was isolated from Coniothyrium sp.-an endophytic fungus isolated from Salsola oppositifolia [42]. The TDDFT ECD calculations were performed using the TZVP basis set and three functionals (B3LYP, BH\&HLYP, CAM-B3LYP) and all of them produced ECD spectra that agreed well with the experimental ECD spectrum; the use of CAM-B3LYP gave the best agreement. Thus, the $\mathrm{AC}$ of $\mathbf{2 4}$ was assigned to be as shown in Fig. 20.

Others

Phomopsinones A-D [43]

Phomopsinones A-D (25-28) were obtained from an endophytic strain of Phomopsis sp., isolated from the halotolerant plant Santolina chamaecyparissus [43]. Hussain and coworkers used (S)-4-methoxy-7-methyl-7,8dihydropyrano[4,3-b]pyran-2(5H)-one (29) as a model compound to find the best functional and basis set level for ECD calculations. The DFT optimized geometry [B3LYP/ 6-31G(d)] with equatorial 7-methyl was used as input in TDDFT ECD calculations with various combinations of hybrid functionals (CAM-B3LYP, B3LYP, PBE0) and basis sets (SVP, TZVP, aug-TZVP). The calculated ECD spectra for $\mathbf{2 9}$ were then compared with the experimental ECD spectrum of 25. The long-range functional CAMB3LYP gave the best agreement, and all the basis sets performed similarly. Thus, the CAM-B3LYP/SVP combination was employed in subsequent ECD calculations of 25, 26, and 28 to assign their ACs to be as shown in Fig. 21.

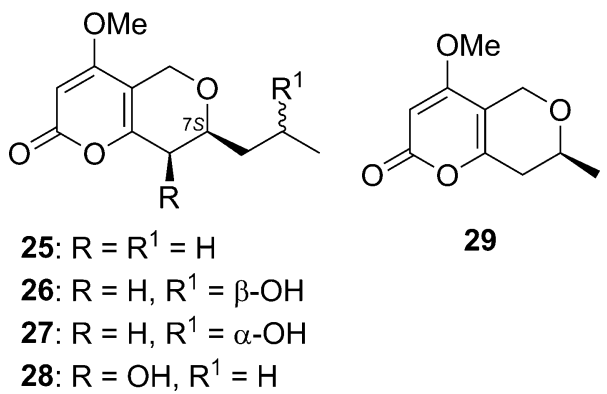

Fig. 21 Structures of phomopsinones A-D (25-28) and $(S)-4-$ methoxy-7-methyl-7,8-dihydropyrano[4,3-b]pyran-2(5H)-one (29) 


\section{Conclusion}

TDDFT calculation of ECD simplified the interpretation of the ECD-AC relationship and is a promising tool for the AC determination of natural products with chiral centers. Successful application of TDDFT calculations of ECD spectra to the AC determination of various type of natural products, ranging from conformationally rigid to highly flexible compounds, are exemplified in this review. Further improvements both in the computer system technologies and TDDFT, may make the TDDFT calculation of ECD an integral part of the $\mathrm{AC}$ determination of natural products.

Acknowledgment Our isolation work was supported by a Grant-inAid for Scientific Research from the Ministry of Education, Culture, Sports, Science, and Technology of Japan.

\section{References}

1. Brickell WS, Mason SF, Roberts DR (1971) $[\pi]$-SCF studies of the circular dichroism and electronic spectra of alkaloids containing the aniline chromophore. The stereochemical configuration of calycanthine and caracurine-II. J Chem Soc B 1971:691-695

2. Li X-C, Ferreira D, Ding Y (2010) Determination of absolute configuration of natural products. Curr Org Chem 14:1678-1697

3. Diedrich C, Grimme S (2003) Systematic investigation of modern quantum chemical methods to predict electronic circular dichroism spectra. J Phys Chem A 107:2524-2539

4. Stephens PJ, McCann DM, Butkus E, Stoncius S, Cheeseman JR, Frisch MJ (2004) Determination of absolute configuration using concerted ab initio DFT calculations of electronic circular dichroism and optical rotation: bicyclo[3.3.1]nonane diones. J Org Chem 69:1948-1958

5. Stephens PJ, McCann DM, Devlin FJ, Cheeseman JR, Frisch MJ (2004) Determination of the absolute configuration of $[32](1,4)$ barrelenophanedicarbonitrile using concerted timedependent density functional theory calculations of optical rotation and electronic circular dichroism. J Am Chem Soc 126:7514-7521

6. Schühly W, Crockett SL, Fabian WMF (2005) Hyperolactone C: determination of its absolute configuration by comparison of experimental and calculated CD spectra. Chirality 17:250-256

7. McCann DM, Stephens PJ (2006) Determination of absolute configuration using density functional theory calculations of optical rotation and electronic circular dichroism: chiral alkenes. J Org Chem 71:6074-6098

8. Crawford TD, Tam MC, Abrams ML (2007) The current state of ab initio calculations of optical rotation and electronic circular dichroism spectra. J Phys Chem A 111:12057-12068

9. Stephens PJ, Devlin FJ, Gasparrini F, Ciogli A, Spinelli D, Cosimelli B (2007) Determination of the absolute configuration of a chiral oxadiazol-3-one calcium channel blocker, resolved using chiral chromatography, via concerted density functional theory calculations of its vibrational circular dichroism, electronic circular dichroism, and optical rotation. J Org Chem 72:4707-4715

10. Bringmann G, Bruhn T, Maksimenka K, Hemberger Y (2009) The assignment of absolute stereostructures through quantum chemical circular dichroism calculations. Eur J Org Chem 2009:2717-2727
11. "Gaussian09." from http://www.gaussian.com

12. “TURBOMOLE 6.3." from http://www.turbomole.com

13. "NWChem 6.0." from http://www.nwchem-sw.org

14. Berova N, Bari LD, Pescitelli G (2007) Application of electronic circular dichroism in configurational and conformational analysis of organic compounds. Chem Soc Rev 36:914-931

15. Pecul M, Ruud K, Helgaker T (2004) Density functional theory calculation of electronic circular dichroism using London orbitals. Chem Phys Lett 388:110-119

16. Klamt A, Schuurmann G (1993) COSMO: a new approach to dielectric screening in solvents with explicit expressions for the screening energy and its gradient. J Chem Soc Perkin Trans 2:799-805

17. Klamt A (1995) Conductor-like screening model for real solvents: a new approach to the quantitative calculation of solvation phenomena. J Phys Chem 99:2224-2235

18. Stephens PJ, Harada N (2010) ECD cotton effect approximated by the Gaussian curve and other methods. Chirality 22:229-233

19. Pedersen TB, Koch H, Ruud K (1999) Coupled cluster response calculation of natural chiroptical spectra. J Chem Phys 110:2883-2892

20. Petrovic AG, Polavarapu PL (2007) Chiroptical spectroscopic determination of molecular structures of chiral sulfinamides: t-butanesulfinamide. J Phys Chem A 111:10938-10943

21. Petrovic AG, Polavarapu PL, Drabowicz J, Lyżwa P, Mikolajczyk M, Wieczorek W, Balinska A (2008) Diastereomers of $N$ - $\alpha$-phenylethyl-t-butylsulfinamide: absolute configurations and predominant conformations. J Org Chem 73:3120-3129

22. Petrovic AG, Vick SE, Polavarapu PL (2008) Determination of the absolute stereochemistry of chiral biphenanthryls in solution phase using chiroptical spectroscopic methods: 2,2'-diphenyl[3,3'-biphenanthrene]-4,4'-diol. Chirality 20:501-510

23. Deguchi J, Shoji T, Nugroho AE, Hirasawa Y, Hosoya T, Shirota O, Awang K, Hadi AHA, Morita H (2010) Eucophylline, a tetracyclic vinylquinoline alkaloid from Leuconotis eugenifolius. J Nat Prod 73:1727-1729

24. Motegi M, Nugroho AE, Hirasawa Y, Arai T, Hadi AHA, Morita H (2012) Leucomidines A-C, novel alkaloids from Leuconotis griffithii. Tetrahedron Lett 53:1227-1230

25. He F, Nugroho AE, Wong CP, Hirasawa Y, Shirota O, Morita H, Aisa HA (2012) Rupestines F-M, new guaipyridine sesquiterpene alkaloids from Artemisia rupestris. Chem Pharm Bull 60:213-218

26. Hirasawa Y, Hara M, Nugroho AE, Sugai M, Zaima K, Kawahara N, Goda Y, Awang K, Hadi AHA, Litaudon M, Morita H (2010) Bisnicalaterines $\mathrm{B}$ and $\mathrm{C}$, atropisomeric bisindole alkaloids from Hunteria zeylanica, showing vasorelaxant activity. J Org Chem 75:4218-4223

27. Stephens PJ, Pan J-J, Devlin FJ, Urbanová M, Hájíček J (2007) Determination of the absolute configurations of natural products via density functional theory calculations of vibrational circular dichroism, electronic circular dichroism and optical rotation: the schizozygane alkaloid schizozygine. J Org Chem 72:2508-2524

28. Renner U, Kernweisz P (1963) Alkaloide aus Schizozygia caffaeoides (Boj.) Baill. Experientia 19:244-246

29. Taniguchi T, Martin CL, Monde K, Nakanishi K, Berova N, Overman LE (2009) Absolute configuration of actinophyllic acid as determined through chiroptical data. J Nat Prod 72:430-432

30. Carroll AR, Hyde E, Smith J, Quinn RJ, Guymer G, Forster PI (2005) Actinophyllic acid, a potent indole alkaloid inhibitor of the coupled enzyme assay carboxypeptidase U/Hippuricase from the leaves of Alstonia actinophylla (Apocynaceae). J Org Chem 70:1096-1099

31. Almeida C, Hemberger Y, Schmitt SM, Bouhired S, Natesan L, Kehraus S, Dimas K, Gütschow M, Bringmann G, König GM (2012) Marilines A-C: novel phthalimidines from the spongederived fungus Stachylidium sp. Chem Eur J 18:8827-8834 
32. Xue M, Zhang Q, Gao J-M, Li H, Tian J-M, Pescitelli G (2012) Chaetoglobosin $\mathrm{Vb}$ from endophytic Chaetomium globosum: absolute configuration of chaetoglobosins. Chirality 24:668-674

33. Najmuldeen IA, Hadi AHA, Awang K, Mohamad K, Ketuly KA, Mukhtar MR, Chong S-L, Chan G, Nafiah MA, Weng NS, Shirota O, Hosoya T, Nugroho AE, Morita H (2011) Chisomicines A-C, limonoids from Chisocheton ceramicus. J Nat Prod 74:1313-1317

34. Nagakura Y, Nugroho AE, Hirasawa Y, Hosoya T, Rahman A, Kusumawati I, Zaini N, Morita H (2013) Sanjecumins A and B: new limonoids from Sandoricum koetjape. J Nat Med 67:381-385

35. Kurtán T, Jia R, Li Y, Pescitelli G, Guo Y-W (2012) Absolute configuration of highly flexible natural products by the solid-state ECD/TDDFT method: ximaolides and sinulaparvalides. Eur $\mathbf{J}$ Org Chem 2012:6722-6728

36. Jia R, Guo Y-W, Chen P, Yang Y-M, Mollo E, Gavagnin M, Cimino G (2007) Biscembranoids and their probable biogenetic precursor from the Hainan soft coral Sarcophyton tortuosum. J Nat Prod 70:1158-1166

37. Jia R, Guo Y-W, Mollo E, Gavagnin M, Cimino G (2008) Further new bis-cembranoids from the Hainan soft coral Sarcophyton tortuosum. Helv Chim Acta 91:2069-2074

38. Xu Y-J, Foubert K, Dhooghe L, Lemière F, Maregesi S, Coleman CM, Zou Y, Ferreira D, Apers S, Pieters L (2012) Rapid isolation and identification of minor natural products by LC-MS, LCSPE-NMR and ECD: isoflavanones, biflavanones and bisdihydrocoumarins from Ormocarpum kirkii. Phytochemistry 79:121-128

39. Dhooghe L, Maregesi S, Mincheva I, Ferreira D, Marais JPJ, Lemière $\mathrm{F}$, Matheeussen $\mathrm{A}, \operatorname{Cos} \mathrm{P}$, Maes L, Vlietinck A, Apers S, Pieters L (2010) Antiplasmodial activity of (I-3, II-3)-biflavonoids and other constituents from Ormocarpum kirkii. Phytochemistry 71:785-791

40. DeAngelis GG, Wildman WC (1969) Circular dichroism studies-I: a quadrant rule for the optically active aromatic chromophore in rigid polycyclic systems. Tetrahedron 25:5099-5112

41. Debbab A, Aly AH, Edrada-Ebel R, Wray V, Pretsch A, Pescitelli G, Kurtan T, Proksch P (2012) New anthracene derivativesstructure elucidation and antimicrobial activity. Eur J Org Chem 2012:1351-1359

42. Sun P, Huo J, Kurtán T, Mándi A, Antus S, Tang H, Draeger S, Schulz B, Hussain H, Krohn K, Pan W, Yi Y, Zhang W (2013) Structural and stereochemical studies of hydroxyanthraquinone derivatives from the endophytic fungus Coniothyrium sp. Chirality $25: 141-148$

43. Hussain H, Krohn K, Ahmed I, Draeger S, Schulz B, Di Pietro S, Pescitelli G (2012) Phomopsinones A-D: four new pyrenocines from endophytic fungus Phomopsis sp. Eur J Org Chem 2012:1783-1789 